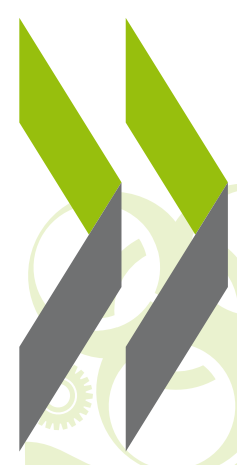

\title{
Compétences et situation sur le marché du travail de la population issue de l'immigration
}




\section{LES COMPÉTENCES DES ADULTES}

\section{ÀLA LOUPE}

Les travailleurs nés à l'étranger sont moins bien rémunérés que leurs homologues autochtones. Ils sont également plus susceptibles d'exercer des professions moins qualifiées et moins prestigieuses.

- La situation défavorable sur le marché du travail des travailleurs nés à l'étranger peut s'expliquer en grande partie par leurs antécédents, en particulier le pays où ils ont fait des études, ainsi que par leur maîtrise de la langue du pays d'accueil et leurs compétences en littératie et en numératie.

Les adultes nés à l'étranger estiment que leurs compétences sont sous-exploitées dans certains pays, ce qui a d'importantes répercussions sur la réussite de leur intégration socio-économique.

Dans les pays de l'OCDE, les individus issus de l'immigration ont un niveau de compétence en littératie et en numératie plus faible que leurs homologues autochtones. L'Évaluation des compétences des adultes (PIAAC) montre que l'écart de niveau de compétence en littératie entre ces deux groupes s'établit à environ 24 points, soit environ 3.5 années de scolarisation (OCDE, 2017). Cette différence peut s'expliquer en grande partie par la maîtrise de la langue du pays d'accueil et par le lieu où les individus issus de l'immigration ont obtenu leur plus haut niveau de qualification. Néanmoins, les compétences en littératie des adultes nés à l'étranger restent en deçà de celles des adultes autochtones même lorsqu'ils ont suivi leurs études dans le pays d'accueil (voir le graphique 1). Ce numéro de la série «Les compétences des adultes à la loupe» fournit de nouveaux éléments probants sur les répercussions sur le marché du travail de ces différences de niveau de compétence entre les deux groupes, et examine le lien entre les compétences des travailleurs nés à l'étranger et leur situation sur le marché du travail.

Graphique 1 / Répartition des résultats des évaluations en littératie et en numératie, selon le statut au regard de l'immigration

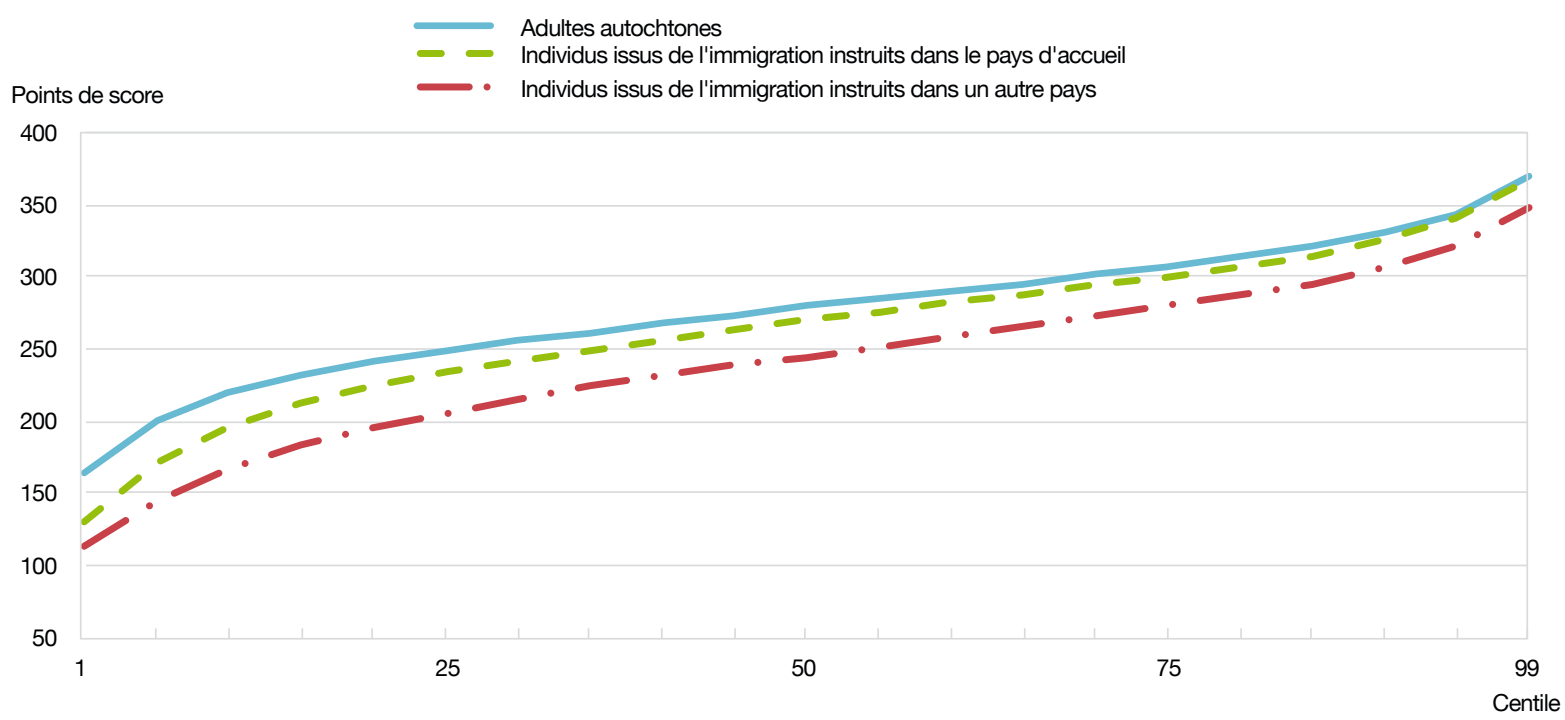

Remarque : Une différence de cinquante points en littératie correspond à environ un écart-type dans la répartition globale des résultats. Source : Évaluation des compétences des adultes (PIAAC) (2012, 2015), www.oecd.org/skills/piaac/data/. 


\section{Les individus issus de l'immigration ont une rémunération beaucoup moins élevée que les adultes autochtones}

Les adultes nés à l'étranger ont souvent un niveau de formation supérieur à celui des adultes autochtones. Par exemple, les personnes issues de l'immigration sont plus susceptibles, en comparaison avec les adultes autochtones, d'être titulaires d'un diplôme universitaire en Amérique du Nord, en Australie, au Danemark, en Irlande, en Norvège, en Nouvelle-Zélande, à Singapour et en Suède. Toutefois, en moyenne, la rémunération horaire des travailleurs autochtones est $5 \%$ supérieure de 5 points à celle des individus issus de l'immigration titulaires d'une qualification obtenue dans le pays d'accueil, et $16 \%$ supérieure à celle des personnes issues de l'immigration qui ont obtenu une qualification à l'étranger (voir le graphique 2). C'est aux États-Unis que l'écart de rémunération est le plus marqué : les adultes nés à l'étranger qui ont suivi leurs études dans un autre pays ne gagnent que $72 \%$ de la rémunération horaire de leurs homologues autochtones. Cet écart de rémunération parmi les personnes issues de l'immigration persiste même lorsque le niveau de formation est pris en compte.

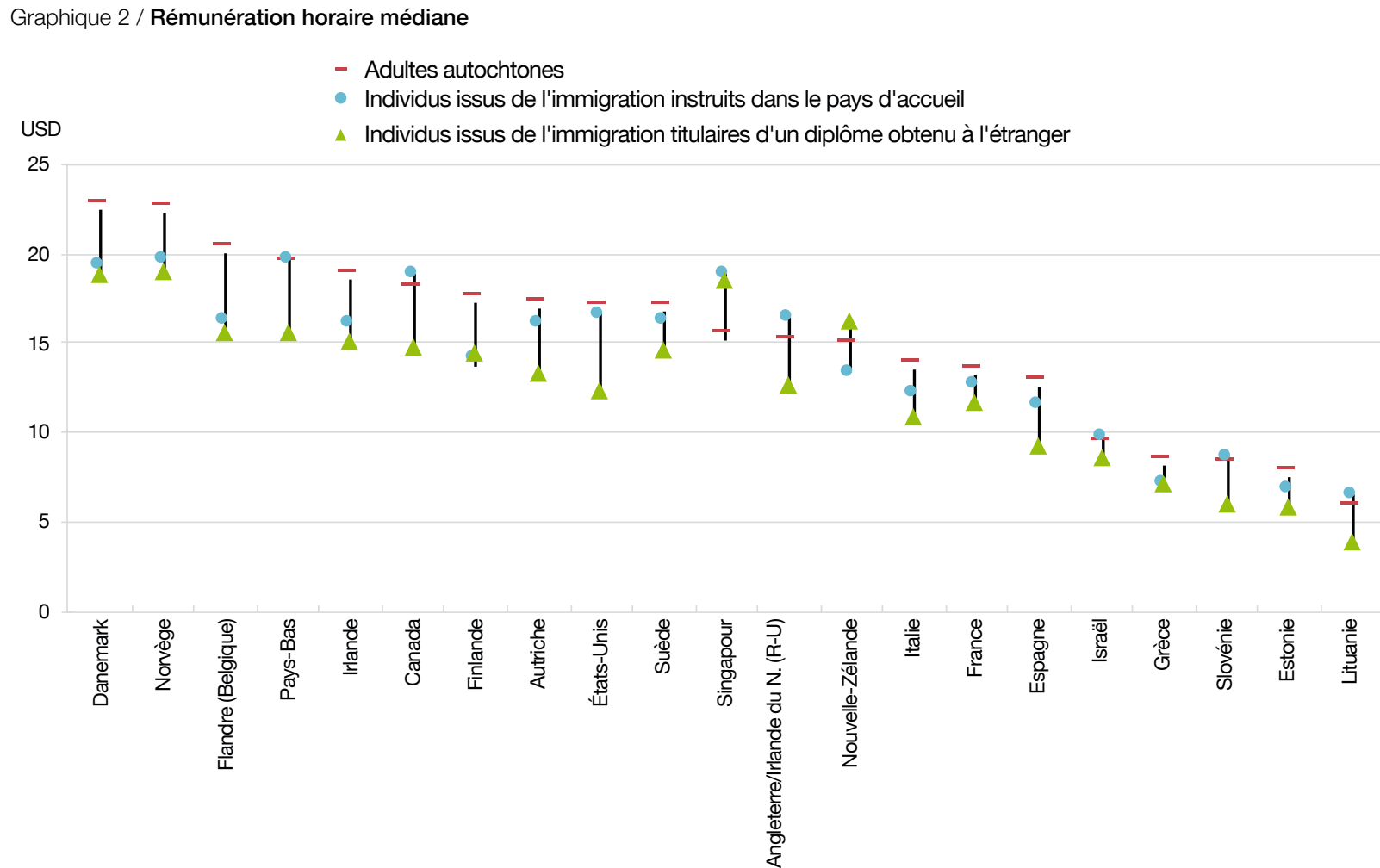

Les pays et économies sont classés par ordre décroissant de la rémunération horaire médiane des personnes autochtones. Source : Évaluation des compétences des adultes (PIAAC) (2012, 2015), www.oecd.org/skills/piaac/data/.

\section{Les adultes nés à l'étranger exercent souvent des professions moins qualifiées et moins prestigieuses}

Cet écart de rémunération s'explique en partie par la différence entre les professions exercées par les travailleurs nés à l'étranger et celles des travailleurs autochtones. Les individus issus de l'immigration sont souvent plus nombreux à occuper des emplois qui ne nécessitent pas un niveau élevé de formation. Dans l'ensemble, les adultes nés à l'étranger qui n'ont pas reçu leur instruction dans le pays d'accueil sont deux fois plus 


\section{LES COMPÉTENCES DES ADULTES}

À LA LOUPE

susceptibles, par comparaison avec leurs homologues autochtones, d'exercer des professions peu qualifiées (voir le graphique 3). Dans certains pays, tels que le Canada, l'Italie et la Nouvelle-Zélande, les individus issus de l'immigration qui ont obtenu leurs qualifications à l'étranger sont cinq fois plus susceptibles, par comparaison avec les adultes nés dans le pays d'accueil, d'exercer un emploi faiblement qualifié.

Toutefois, ce sont les compétences et la maîtrise de la langue employée dans l'enquête PIAAC qui expliquent en grande partie ces différences. Ce constat est particulièrement vrai pour les travailleurs diplômés de l'enseignement tertiaire. Lorsque les caractéristiques individuelles (telles que le sexe et l'âge) et les compétences sont prises en compte, les adultes nés à l'étranger et les adultes autochtones diplômés de l'enseignement tertiaire sont tout autant susceptibles d'occuper un emploi hautement qualifié. En d'autres termes, toutes choses étant égales par ailleurs, si les travailleurs diplômés de l'enseignement tertiaire ont tous le même niveau de compétences, leur pays d'origine n'a pas d'incidence sur leur probabilité d'exercer un emploi plus prestigieux ${ }^{1}$. Par contraste, les compétences et le niveau de maîtrise de la langue nationale font partie des aspects qui ont un rôle moins important, mais qui reste néanmoins significatif pour expliquer les différences professionnelles entre les individus moins instruits, tandis que d'autres facteurs qui n'ont pas été observés (tels que les réseaux, la discrimination et les asymétries d'information) jouent également un rôle décisif à cet égard.

Graphique 3 / Différences relatives à l'utilisation des compétences en lecture dans le cadre professionnel entre les adultes nés à l'étranger et les adultes autochtones

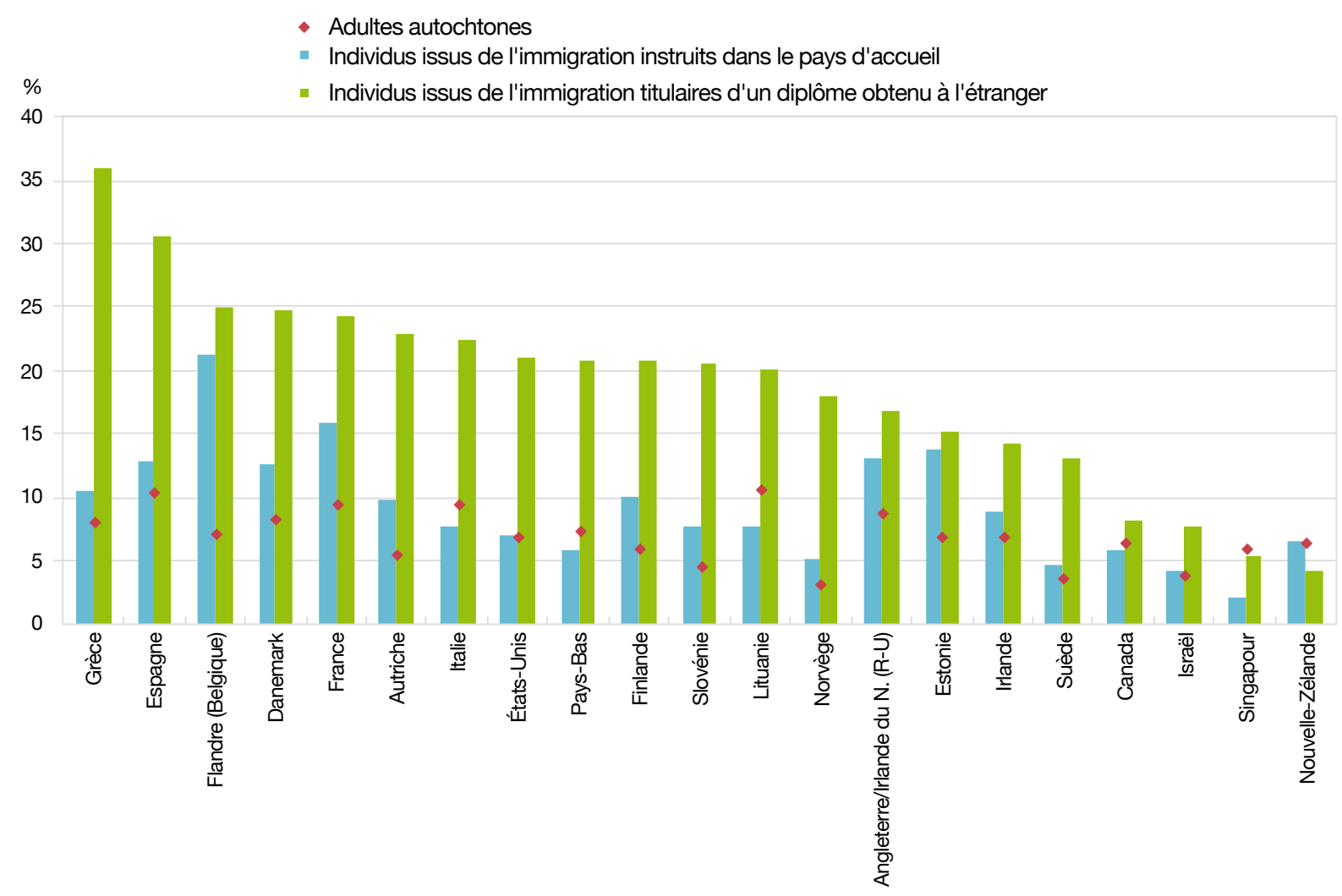

Remarque : Les professions peu qualifiées correspondent aux professions relevant de la catégorie 9 de la CITP, c'est-à-dire les « professions élémentaires ». Les pays et économies sont classés par ordre décroissant du pourcentage de travailleurs nés à l'étranger qui ont obtenu leurs qualifications à l'étranger et qui exercent des professions peu qualifiées.

Source : Évaluation des compétences des adultes (PIAAC) (2012, 2015), www.oecd.org/skills/piaac/data/. 
Dans plusieurs pays, les personnes issues de l'immigration indiquent que leurs compétences sont sous-exploitées (voir le graphique 4). Par exemple, par comparaison avec les adultes nés dans le pays, les adultes nés à l'étranger qui ont suivi leurs études en Suède sont plus susceptibles (à hauteur de 4 points de pourcentage) de se sentir surqualifiés pour l'emploi qu'ils exercent, et ceux qui ont suivi leurs études à l'étranger le sont encore plus (à hauteur de 8 points de pourcentage). Des constats similaires s'observent au Danemark et à
Singapour. Cette inadéquation des compétences déclarée par les individus est fondamentale pour l'intégration socio-économique des personnes issues de l'immigration, puisqu'elle a non seulement une incidence sur leur rémunération mais aussi sur leur niveau général de satisfaction et de bien-être. Ces inadéquations subjectives montrent que le marché du travail ne parvient pas à tirer pleinement parti des capacités des personnes issues de l'immigration, ce qui se solde par une diminution des opportunités économiques.

\section{Graphique 4 / Pourcentage de travailleurs qui estiment que leurs compétences sont sous-exploitées}

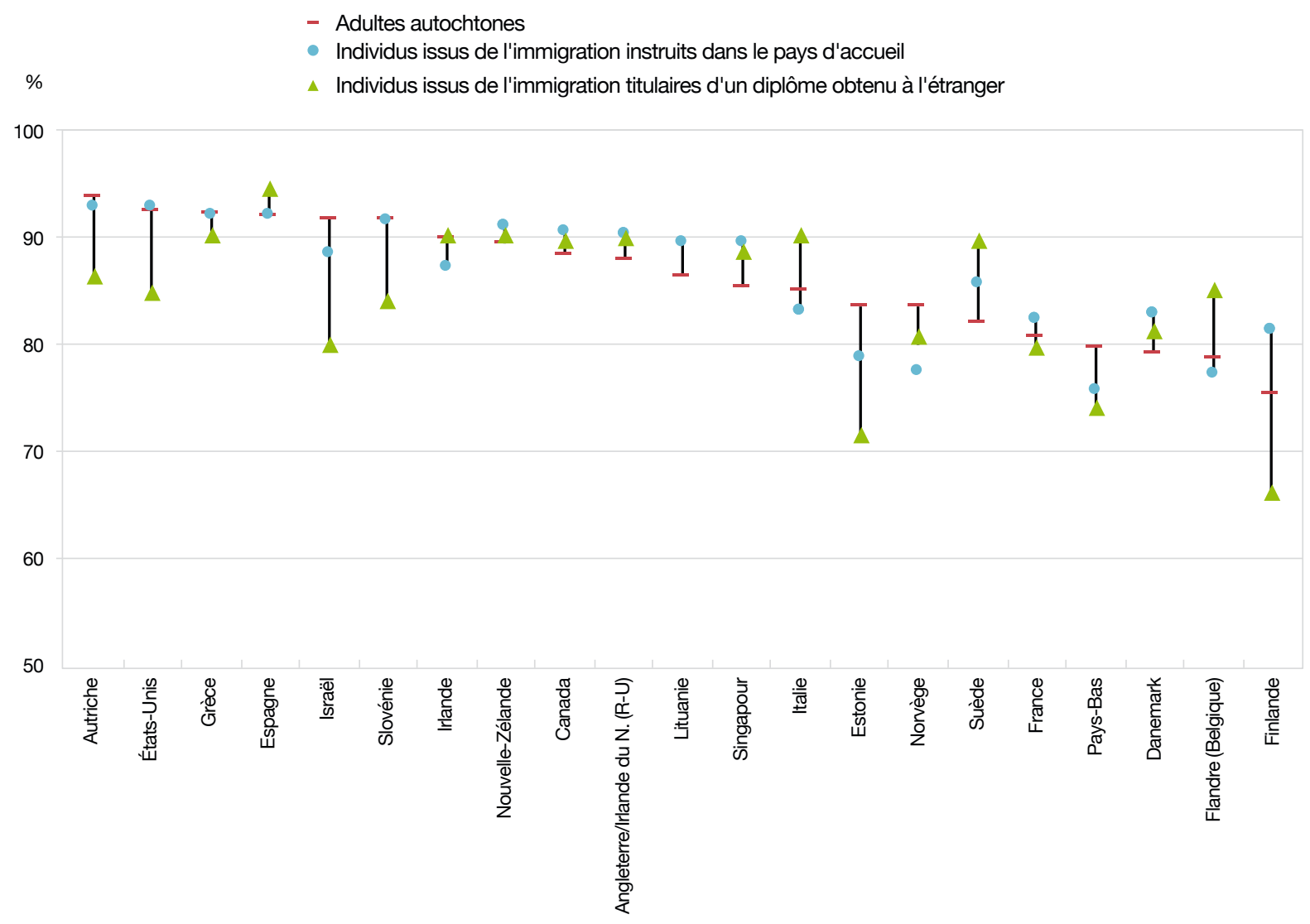

Les pays et économies sont classés par ordre décroissant du pourcentage de travailleurs autochtones estimant que leurs compétences sont sous-exploitées. Source : Évaluation des compétences des adultes (PIAAC) (2012, 2015), www.oecd.org/skills/piaac/data/. 


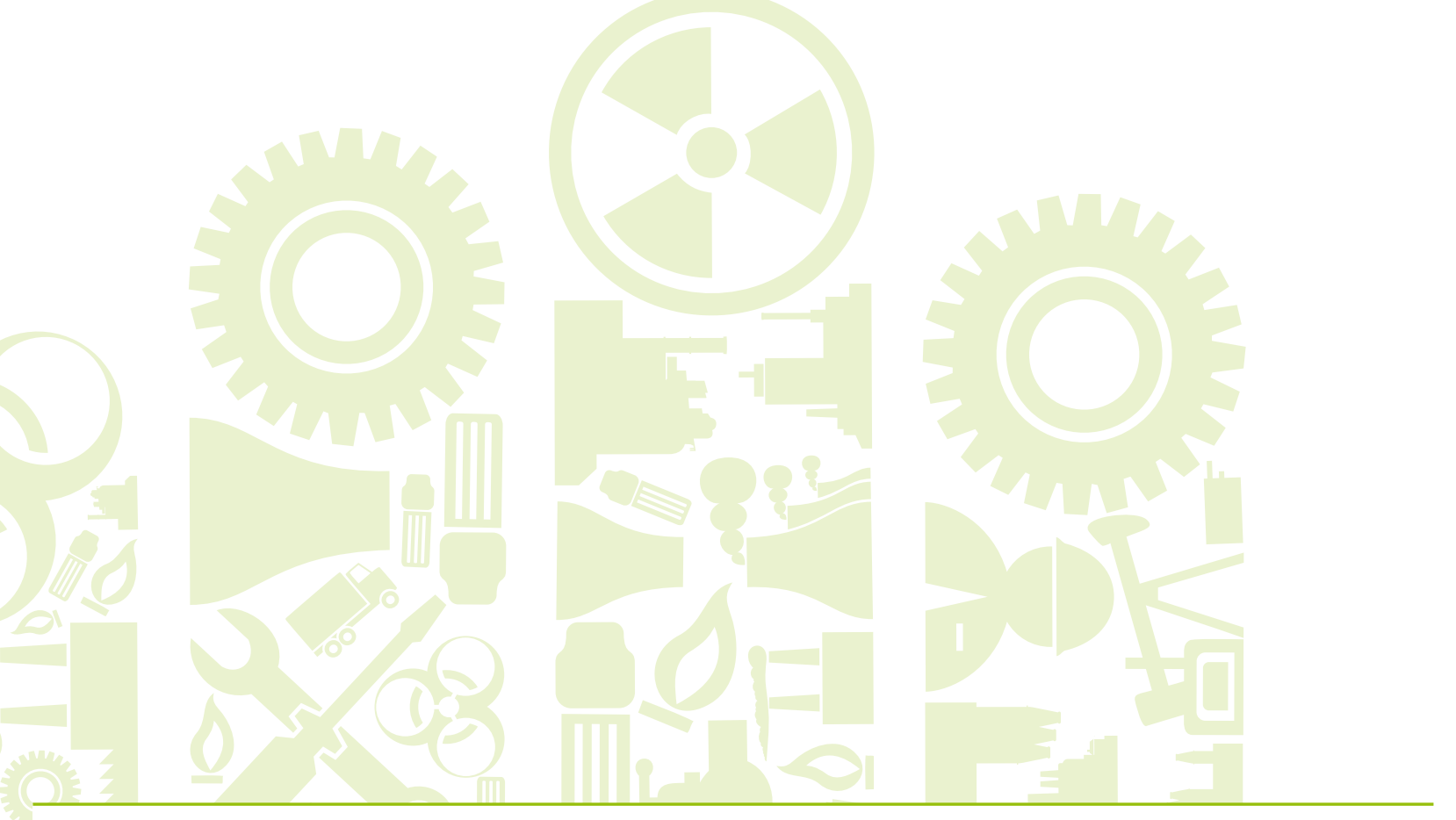

Dans l'OCDE, la situation sur le marché du travail des adultes nés à l'étranger est généralement moins favorable que celle des adultes autochtones. Les personnes issues de l'immigration sont non seulement plus susceptibles d'être sans emploi ou inactives, mais elles sont également plus susceptibles d'être moins bien rémunérées et d'exercer des emplois moins qualifiés. Les différences liées à leurs compétences, à leur maîtrise de la langue parlée et au pays dans lequel elles ont été instruites expliquent en grande partie, bien que pas totalement, les écarts qui sont observés. II convient par conséquent de prévoir des programmes efficaces d'enseignement et de formation pour les adultes et des cours de langues pour les individus issus de l'immigration afin de garantir la réussite de l'intégration des travailleurs nés à l'étranger au sein des économies d'accueil.

\section{> CONTACT : $\quad$ Michele Tuccio (michele.tuccio@oecd.org); (edu.piaac@oecd.org) \\ > POUR EN OECD (2018), Skills on the Move: Migrants in the Survey of Adult Skills, \\ SAVOIR PLUS : Études de l'OCDE sur les compétences, OECD Publishing, Paris, https://doi.org/10.1787/9789264307353-en}

OECD (2017), «Pourquoi les adultes immigrés sont-ils moins compétents en littératie que leurs pairs autochtones ?", Les compétences des adultes à la loupe, No. 6, OECD Publishing, Paris, https://doi.org/10.1787/bc5c81e1-fr.

$>$ VISITER : www.oecd.org/skills/piaac

Indicateurs de l'éducation à la loupe - PISA à la loupe - L'enseignement à la loupe

L'Évaluation des compétences des adultes est un produit du Programme de l'OCDE pour l'évaluation internationale des compétences des adultes (PIAAC).

Ce document est publié sous la responsabilité du Secrétaire général de l'OCDE. Les opinions qui y sont exprimées et les arguments qui y sont employés ne reflètent pas nécessairement les vues officielles des pays membres de l'OCDE.

Ce document et toute carte qu'il peut comprendre sont sans préjudice du statut de tout territoire, de la souveraineté s'exerçant sur ce dernier, du tracé des frontières et limites internationales, et du nom de tout territoire, ville ou région.

Vous êtes autorisés à copier, télécharger ou imprimer du contenu OCDE pour votre utilisation personnelle. Vous pouvez inclure des extraits des publications, des bases de données et produits multimédia de l'OCDE dans vos documents, présentations, blogs, sites Internet et matériel d'enseignement, sous réserve de faire mention de la source OCDE et du copyright. Les demandes pour usage public ou commercial ou de traduction devront être adressées à pubrights@oecd.org.

Les données statistiques concernant Israël sont fournies par et sous la responsabilité des autorités israéliennes compétentes. L'utilisation de ces données par l'OCDE est sans préjudice du statut des hauteurs du Golan, de Jérusalem Est et des colonies de peuplement israéliennes en Cisjordanie aux termes du droit international. 